\title{
Nonlocal Electron-Phonon Interaction as a Source of Dynamic Charge Stripes in the Cuprates
}

\author{
Claus Falter and Thomas Bauer \\ Institut für Festkörpertheorie, Westfälische Wilhelms-Universität, Wilhelm-Klemm-Straße 10, 48149 Münster, Germany \\ Correspondence should be addressed to Claus Falter, falter@uni-muenster.de
}

Received 14 March 2012; Accepted 20 September 2012

Academic Editor: Jörg Fink

Copyright ( 2012 C. Falter and T. Bauer. This is an open access article distributed under the Creative Commons Attribution License, which permits unrestricted use, distribution, and reproduction in any medium, provided the original work is properly cited.

\begin{abstract}
We calculate for $\mathrm{La}_{2} \mathrm{CuO}_{4}$ the phonon-induced redistribution of the electronic charge density in the insulating, the underdoped pseudogap, and the more conventional metallic state as obtained for optimal and overdoping, respectively. The investigation is performed for the anomalous high-frequency oxygen bond-stretching modes, which experimentally are known to display a strong softening of the frequencies upon doping in the cuprates. This most likely generic anomalous behaviour of these modes is shown to be due to a strong nonlocal electron-phonon interaction mediated by charge fluctuations on the ions. We demonstrate that the softening of the modes is caused by nonlocally induced dynamic charge inhomogeneities in form of charge stripes along the $\mathrm{CuO}$ bonds with different orbital character. The dynamic charge inhomogeneities may in turn be considered as precursors of static charge stripe order as recently observed in $\mathrm{La}_{2-x} \mathrm{Ba}_{x} \mathrm{CuO}_{4}$ in a broad range of doping around $x=1 / 8$. The latter may trigger a reconstruction of the Fermi surface into small pockets with reduced doping. We argue that the incompressibility of the Cu3d orbital and simultaneously the compressibility of the $\mathrm{O} 2 p$ orbital in the pseudogap state seem to be required to nucleate dynamic stripes.
\end{abstract}

In recent work $[1,2]$, we have developed a description of the electronic charge response and phonon dynamics for $p$ doped and $n$-doped cuprate high-temperature superconductors (HTSCs). This approach is based upon a microscopic modeling of the change of the charge response across the insulator-metal transition in terms of the compressibility of the electronic system as a primary tool to characterize the corresponding competing ground states depending on doping. This is achieved starting from the insulating state via a metallic pseudogap state to a more conventional metallic state by consecutive orbital selective incompressibilitycompressibility transitions in terms of strict sum rules of the charge response given in [1] and listed below. These sum rules are a proper instrument to describe the competition between the localisation and delocalisation of the electrons, which is at the heart of the physics in the cuprates.

In the conventional metallic state, the electronic partial density of states (PDOS) at the Fermi level $Z_{\kappa}\left(\varepsilon_{F}\right)$ is related to the polarizability matrix in the longwavelength $\operatorname{limit}(\mathbf{q} \rightarrow \overrightarrow{\mathbf{0}})$ according to

$$
\sum_{\kappa^{\prime}} \pi_{\kappa \kappa^{\prime}}(\overrightarrow{\mathbf{q}} \longrightarrow \overrightarrow{\mathbf{0}})=Z_{\kappa}\left(\varepsilon_{F}\right),
$$

and the total density of single particle states at energy $\varepsilon$ is given by

$$
Z(\varepsilon)=\sum_{\kappa} Z_{\kappa}(\varepsilon) .
$$

$\kappa, \kappa^{\prime}$ are the orbital degrees of freedom in the elementary cell of the crystal. In our calculation for $\mathrm{LaCuO}, \mathrm{Cu} 3 d, \mathrm{Cu} 4 s$, and $\mathrm{O} 2 p$ orbitals in the $\mathrm{CuO}$ plane are taken into account. $\mathbf{q}$ is a wavevector from the first Brillouin zone (BZ). On the other hand, for the insulating state we obtain the sum rules

$$
\begin{aligned}
& \sum_{\kappa^{\prime}} \pi_{\kappa \kappa^{\prime}}(\overrightarrow{\mathbf{q}} \longrightarrow \overrightarrow{\mathbf{0}})=O(q), \\
& \sum_{\kappa, \kappa^{\prime}} \pi_{\kappa \kappa^{\prime}}(\overrightarrow{\mathbf{q}} \longrightarrow \overrightarrow{\mathbf{0}})=O\left(q^{2}\right) .
\end{aligned}
$$


The sum $\sum_{\kappa, \kappa^{\prime}} \pi_{\kappa \kappa^{\prime}}(\mathbf{q} \rightarrow \mathbf{0})$ is equal to $\rho^{2} K$ with $\rho$ the average density and $K$ the compressibility of the electronic system. The latter provides a measure of the charge gap in the electronic spectrum because $K$ vanishes as a function of the chemical potential in the gap region. Moreover, $1 / K$ provides a measure of the change of the chemical potential with particle number.

Approaching the delocalization-localization transition from the conventional metallic region representative for optimal and overdoping, when $p$-doping is decreased the $\mathrm{Cu} 3 d$ component of the wave function is admitted to become incompressible, insulator-like in the underdoped state according to our modeling. This means that the correlated $3 \mathrm{~d}$ electrons are allowed to become more localized as compared with the conventional metallic state and disappear from the Fermi surface (FS). Moreover, this effectively models a decoupling of the $3 \mathrm{~d}$ electrons from the holes on the $\mathrm{O} 2 \mathrm{p}$ orbitals quite in contrast to the conventional metallic state. In terms of the sum rule, we then have

$$
\sum_{\kappa^{\prime}} \pi_{\kappa \kappa^{\prime}}(\overrightarrow{\mathbf{q}} \longrightarrow \overrightarrow{\mathbf{0}})= \begin{cases}O(q), & \mathrm{Cu} 3 d \\ Z_{\kappa}\left(\varepsilon_{F}\right), & \mathrm{O} 2 p\end{cases}
$$

Accordingly, the partial density of states is suppressed at the Fermi level for the correlated, localized Cu3d orbitals (orbital intrinsic incompressibility due to correlation) but not for the more delocalized $\mathrm{O} 2 p$ orbitals at the $\mathrm{O}_{x y}$ sublattices in the $\mathrm{CuO}$ plane, where the holes are predominantly injected in the p-type cuprates. So, we have an orbital selective compressible, metallic charge response only for the $\mathrm{O} 2 p$ states with a renormalized PDOS $Z_{\kappa}\left(\varepsilon_{F}\right)$ and a loss in the total density of state at $\varepsilon_{F}$ (pseudogap) because of the incompressibility of the $\mathrm{Cu} 3 d$ states according to (4). The loss in spectral weight also is never recovered on entering the superconducting state. The incompressible regions in the pseudogap state compete with overall metallic behaviour and with superconductivity.

Our modeling of the pseudogap state in terms of compressible, metallic $\mathrm{O} 2 p$ states and $\mathrm{Cu} 3 d$ states treated as incompressible and disappearing from the FS because of strong correlation is supported by calculations performed within the self-interaction-corrected (SIC) local spin density (LSD) approximation for $\mathrm{La}_{2} \mathrm{CuO}_{4}$ [3]. Here it is shown that the self-interaction pulls the occupied localized $\mathrm{Cu} 3 d$ states below the $\mathrm{O} 2 p$ band, only little $\mathrm{Cu} 3 d$ admixture is found in the $\mathrm{O} 2 p$ band.

Furthermore, our modeling of the pseudogap state corresponds with a smaller Fermi volume as compared with the conventional metallic Fermi-liquid state because the Fermi volume is determined by the density of the compressible metallic charge carriers (holes) of dominantly O2p-type alone while the $\mathrm{Cu} 3 d$ states are incompressible as in the Mott insulator and cannot contribute to the Fermi volume quite in contrast to the conventional metallic state where both the $\mathrm{O} 2 p$ and the $\mathrm{Cu} 3 d$ states are compressible metallic leading to a large Fermi volume. The pseudogap state is separated from the conventional overall compressible Fermi-liquid state by an orbital selective compressibility-incompressibility transition of the Cu3d orbital. In the context of our modeling of the pseudogap state, it is interesting to point to another modeling of this state within the framework of the so-called fractionalized Fermi liquid. For a recent discussion see for example, [4] and references therein. In this approach, an idea is important which is based on the concept of an orbitalselective Mott transition. For the latter, for example, in a twoband model, we have one metallic band with Fermi-liquidlike quasiparticles while the other band, for example, related to localized $d$ electrons, undergoes a Mott transition to an insulating state due to strong electronic correlations. Such a transition is analogous to the compressibility-incompressibility transition of the $\mathrm{Cu} 3 d$ orbital in our approach.

Because particle density fluctuations scale with the compressibility of the system, the sum rules introduce varying and orbital dependent fluctuations of the particle number in the different metallic states of the HTSC according to our modeling. The tuning parameter for these fluctuations of the particle number is doping, and the pseudogap state may be considered as a state with reduced particle density fluctuations as compared with the optimal and overdoped state. Moreover, the partial incompressibility of the Cu3d orbitals competes with superconductivity because the phase fluctuations $\Delta \phi$ of the order parameter grow with decreasing particle fluctuations $\Delta N$ due to the uncertainty relation $\Delta N \Delta \phi \sim 1$.

Qualitatively, the incompressibility of the Cu3d states also corresponds to a compartmentalisation of configuration space. This means that some parts of direct space cannot be approached or are hardly accessible to the charge carriers. On the other hand, this should be accompanied by a reciprocal compartmentalisation of momentum space, according to the uncertainty relationship $\Delta x \Delta k \sim 1$. As a consequence, the momentum should have corresponding fluctuations and the FS of the conventional metallic state becomes fuzzy when passing to the underdoped state and possibly reconstructs. ARPES studies and quantum-oscillation measurements seem to reflect this in form of a reconstruction of the large FS in the optimal and overdoped state into small Fermi surface pockets with Fermi-liquid-like charge carriers in the underdoped pseudogap state characterized by a loss of density of states at the Fermi level consistent with our modeling.

In our orbital-based local picture, the pseudogap state of the cuprates looks like a "two-component" electronic structure where a real space organisation of the low lying charge excitations is achieved in form of a metallic charge response by mobile holes on the oxygen network in the $\mathrm{CuO}$ plane that is blocked at the incompressible insulator-like $\mathrm{Cu}$ sites. Inspection of the sum rule in (4) shows that the incompressible $\mathrm{Cu} 3 d$ states and the compressible $\mathrm{O} 2 p$ states are entangled by the off-diagonal elements of the polarizability matrix. So our modelling of the pseudogap state is consistent with a strange not overall compressible metallic state coexisting with atom-like, incompressible $\mathrm{Cu} 3 d$ orbitals same as in the undoped, insulating state favoring short-ranged magnetic spin correlations and corresponding excitations, typically spin fluctuations. The latter should be damped by electron-hole charge excitations of the compressible metallic $\mathrm{O} 2 p$ states. 


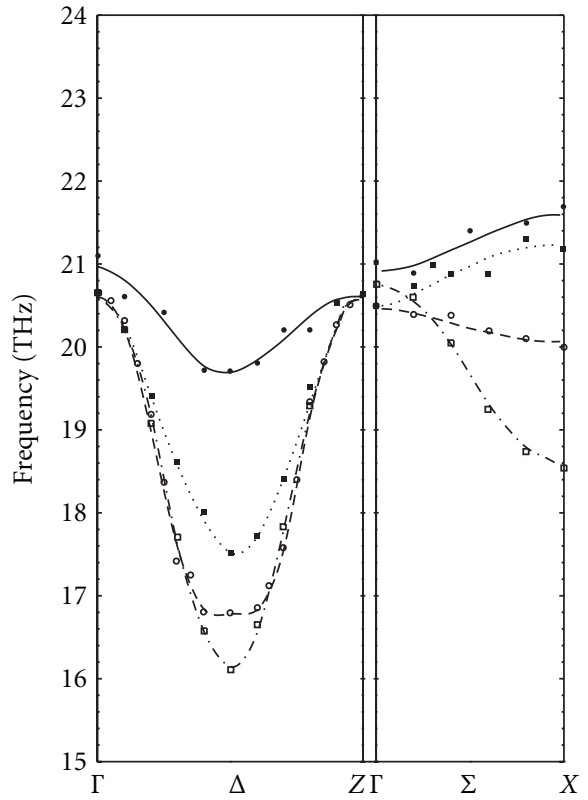

(a) Experimental Results

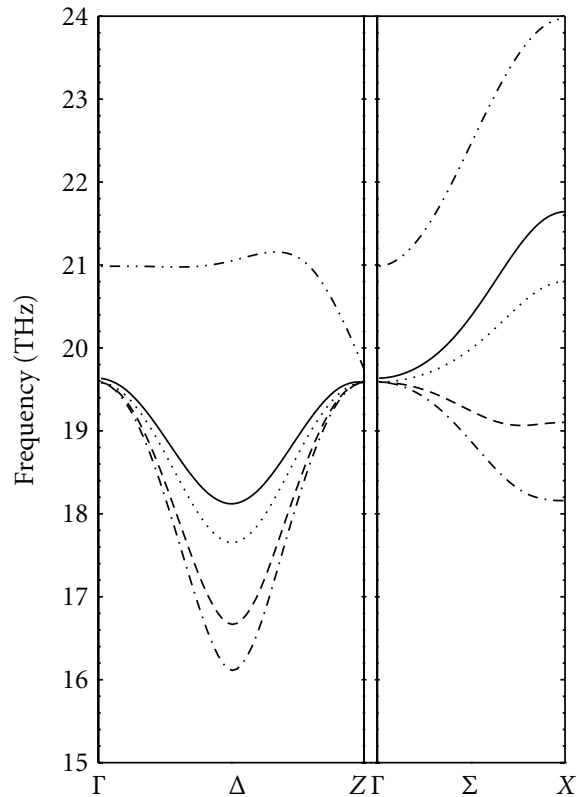

(b) Calculated Results

Figure 1: (a) Experimental results for the highest $\Delta_{1}$ and $\Sigma_{1}$ branch of $\mathrm{La}_{2-x} \mathrm{Sr}_{x} \mathrm{CuO}_{4}$ for various doping levels [6]. •: $x=0.00$, $\mathbf{\square}$ : $x=0.10, \circ: x=0.15, \square: x=0.30$. The lines are a guide to the eye. (b) Calculated results of the phonon branches shown in (a) as obtained with our model approach for the electronic state of the HTSCs [1]. —: insulating state, $\cdots \cdot$ : underdoped state, --- : optimally doped state, $-\cdot-$ : overdoped state. For comparison, the results for the RIM are shown $(-\cdots-)$ in order to demonstrate the crucial influence of the nonlocal EPI effects in form of CFs on the dispersion.

In $k$-space language such a strange mixed locally compressible-incompressible state should result in a heavily renormalized electronic bandstructure and Fermi surface (FS) as compared with the more conventional overall compressible Fermi liquid state with a large FS at higher doping. The pseudogap state displaying properties characteristic of both insulators and correlated metals evolves from the incompressible insulating state with no low energy charge excitations and seems to promote the tendency to charge and spin ordering that breaks rotational and translational symmetry, for example, in form of stripes. The latter appear to be quite common in underdoped cuprates. In this context it is interesting to point out that the stripe-like modulation observed in [5] is strongest on the oxygen orbitals. So, the latter are very essential to understand the physics of charge order in the cuprates and also the charge response as shown below. Finally, the enhanced phase fluctuations due to the incompressibility of the $\mathrm{Cu} 3 d$ orbital favour pairing correlations in the normal state, possibly incoherent preformed pairs as discussed in the literature.

Our modeling of the density response is strongly supported by corresponding calculations of phonon dynamics $[1,2]$, which compare well with the experimental data. This is shown for $p$-doped $\mathrm{LaCuO}$ in case of the strongly doping-dependent oxygen bond-stretching mode (OBSM) anomalies, see Figure 1, where the experimental results for the highest $\Delta_{1}$ and $\Sigma_{1}$ branches of $\mathrm{La}_{2-x} \mathrm{Sr}_{x} \mathrm{CuO}_{4}$ are shown for various doping levels [6] and compared with the calculated results. The high-frequency OBSMs are generally known to display an anomalous softening upon doping in the cuprates [7-18]. Such a behaviour of the experimental phonon dispersion is clearly visible for $\mathrm{LaCuO}$ in Figure 1(a) and is very well accounted for by the calculation in our model for the electronic state in terms of incompressibilitycompressibility transitions, see Figure $1(\mathrm{~b})$. We also have included in Figure 1(b) the phonon dispersion of a rigid-ion model (RIM) for $\mathrm{LaCuO}$.

It seems appropriate to explain at least the ideas of our modeling of phonon dynamics and charge response of the cuprates. A quantitative treatment of the theory and the modeling would be too extensive and can be found in $[1,2]$.

The rigid part of the electronic charge response and the electron-phonon interaction (EPI) is approximated by an ab initio rigid-ion model (RIM), taking into account covalent ion softening in terms of (static) effective ionic charges calculated from a tight-binding analysis. In addition, scaling of the short-range part of certain pair potentials between the ions is performed to simulate further covalence effects in the calculation in such a way that the energy-minimized structure is as close as possible to the experimental one. The RIM with the corrections just mentioned then serves as an unbiased reference system for the description of the cuprate superconductors and can be considered as a first approximation for the insulating state of these compounds because of the strong ionic nature of bonding in the cuprates. Starting with such a rigid reference system nonlocal, electronic polarisation processes are introduced in the form of more or less localized electronic charge fluctuations (CF's) at the outer shells of the ions. In the metallic state of the cuprates, especially the latter dominate the nonlocal 


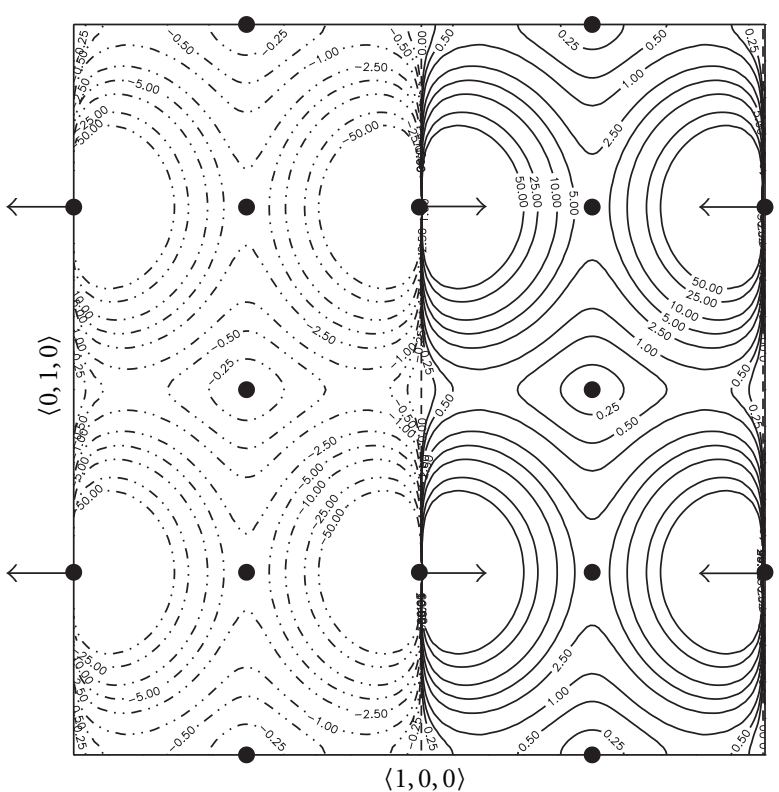

(a)

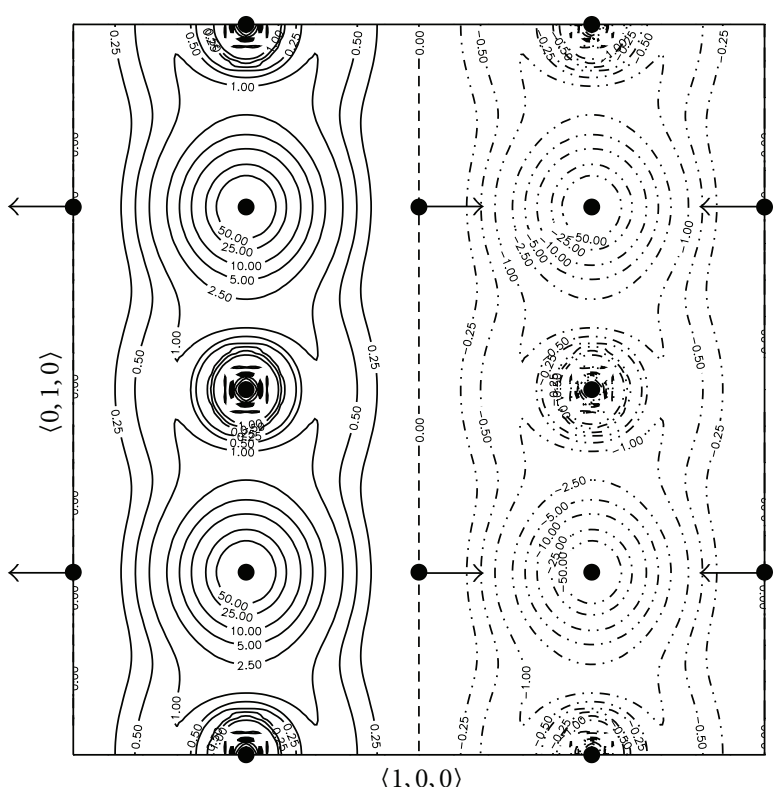

(b)

Figure 2: (a) Contour plot in the $\mathrm{CuO}$ plane of the displacement-induced charge density redistribution $\delta \rho$ for the $\Delta_{1} / 2$ mode within the rigid ion model (RIM) for $\mathrm{La}_{2} \mathrm{CuO}_{4}$. (b) Same as (a), but for the nonlocal part of the charge response in the optimally doped state due to nonlocal EPI effects in the half-breathing mode $\Delta_{1} / 2$. The displaced ions are indicated by a dot with an arrow. Full lines (-) mean that electrons are accumulated in the corresponding region of space and the broken dotted lines $(-\cdots-)$ indicate regions where the electrons are pushed away. $\delta \rho$ is given in units of $10^{-4} e^{2} / a_{B}^{3}$.

contribution of the charge response and the EPI and are particularly important in the $\mathrm{CuO}$ planes. In addition, anisotropic dipole fluctuations are admitted in our approach. They prove to be specifically of interest for the ions in the ionic layers.

Comparing with Figure 1(b), the results of the phonon dispersion of the RIM, where only local EPI effects are considered, with the full calculation we can extract the crucial influence of the nonlocal EPI effects generated by the charge fluctuations. For example, we find a very strong softening of the $\Delta_{1} / 2$ bond-stretching mode (half-breathing mode) increasing with doping up to about $5 \mathrm{THz}$. Comparing the calculated results with the experiments in Figure 1(a), we find a good agreement. In order to achieve such a realistic description of the phonon modes, both, nonlocal EPI effects and multiorbital effects are required. The comparison with the results from the RIM demonstrates that nonlocality is needed and from our detailed calculations we find that the CF's of the more delocalized $\mathrm{O} 2 p$ and Cu4s orbital contribute significantly to the softening of the modes, besides the more localized Cu3d orbital. The strong softening of the phonon anomalies is accompanied by corresponding dynamic charge inhomogeneities in form of charge stripes along the $\mathrm{CuO}$ bonds. The modes are not really soft but one may consider these dynamic stripes as precursors of static charge stripe order with ordering vectors corresponding to the wavevectors of the phonon anomalies.

Finally, it should be remarked that a constructive interplay of electron-ion interaction with short-ranged antiferromagnetic order is important for the $\mathrm{Cu}$ sites in the underdoped and insulating state, because electrons are transferred from the $\mathrm{Cu}$ ion where the $\mathrm{Cu}$ bond is compressed to the $\mathrm{Cu}$ ion where the bond is stretched, see for example, Figure 2. Both ions have opposite spin in the antiferromagnetic case. In contrast if a ferromagnetic order would be present, the Pauli blocking would suppress the transport of electrons with the same spin and consequently also the charge fluctuations and the corresponding softening of the phonon modes.

Modelling of the electronic polarizability $\pi_{\kappa \kappa^{\prime}}(\mathbf{q})$ for the insulating and pseudogap state is needed because a quantitative calculation of $\pi_{\kappa \kappa^{\prime}}(\mathbf{q})$ is not available due to the missing of a rigorous knowledge of this state. On the other hand, for the more conventional metallic state $\pi_{\kappa \kappa^{\prime}}(\mathbf{q})$ can be calculated within the local density approximation (LDA), however, with some important corrections concerning the electronic $c$-axis response which is overestimated in typical LDA-based calculations [19]. Concerning this case it should be remarked that a comparison of the calculated OBSM anomalies from the model in Figure 1(b) with corresponding results obtained within the framework of the corrected LDA [19], taking into account the full $\mathbf{q}$ dependence of the polarizability matrix, leads to a good agreement. This also speaks in favour of our modeling of the charge response in terms of the compressibility sum rules, in which only the longwavelength limit of the polarizability matrix is considered.

In Figure 2 we display the calculated phonon-induced charge redistribution of the bond-stretching mode at $\Delta_{1} / 2$ for the optimal doped state. Figure 2(a) illustrates the effect 


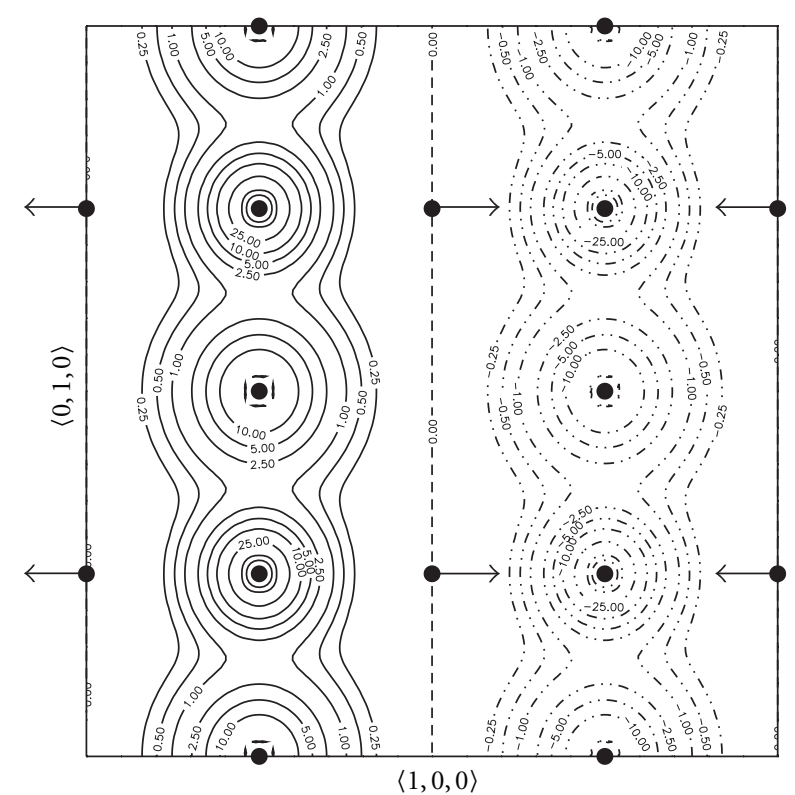

(a)

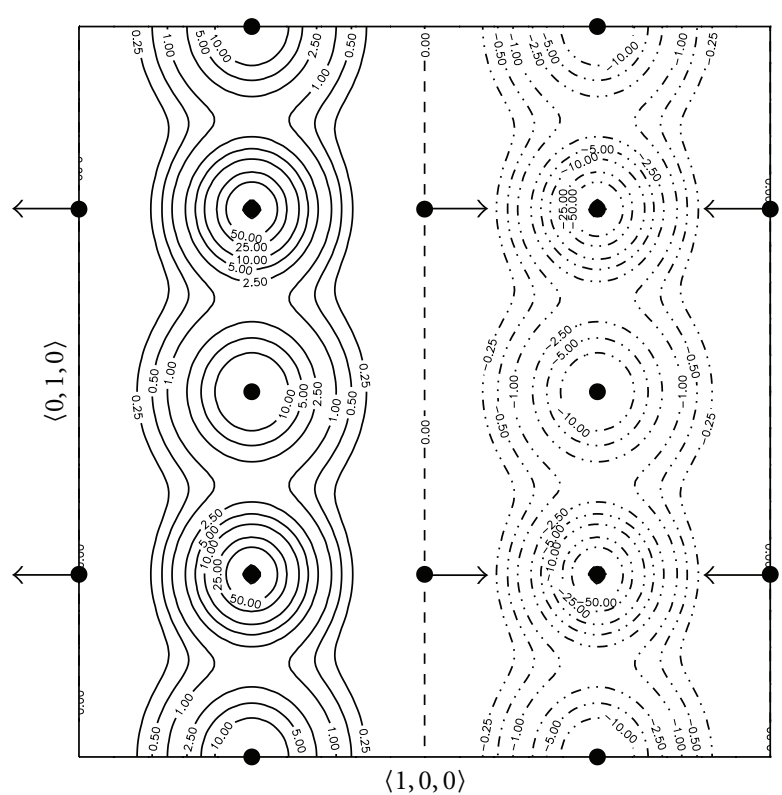

(b)

Figure 3: (a) Same as Figure 2 but for the nonlocal part of the charge response $\delta \rho$ for $\Delta_{1} / 2$ in the insulating state ( $x=0$ ). (b) Same as Figure 2 but for the nonlocal part of the charge response $\delta \rho$ for $\Delta_{1} / 2$ in the pseudogap state $(x=0.1)$.

of the local EPI within the RIM on the charge redistribution connected with the displacement of the $\mathrm{O}_{x}$ ions in the $\mathrm{CuO}$ plane, that is, a stretching or compression of the $\mathrm{CuO}$ bond, respectively, (half-breathing mode (HBM)). The local EPI reveals a charge redistribution of dipole character localized only at the ions as can be expected from the rigid shift of the ionic densities in the RIM during the phonon mode.

From Figure 2(a) we also obtain the message that no dynamical charge stripes are excited by local EPI. On the other hand, Figure 2(b) that displays the nonlocal part of the charge response due to nonlocal EPI effects of charge fluctuation type demonstrates how the strong nonlocal EPI generates dynamic charge stripes in the half-breathing mode. Thus, the moving $\mathrm{O}_{x}$ ions in the HBM, which are linked up with strong CF's on the silent $\mathrm{Cu}$ and $\mathrm{O}_{y}$ ions, induce dynamical stripes in the $y$ direction with a recurrence periond of two lattice constants along the orthogonal $x$ direction. Note, that there are no changes of the transfer integral between $d$ and $p$ orbitals for the silent $\mathrm{Cu}$ and $\mathrm{O}_{y}$ ions, nevertheless there is a charge transfer.

In case of the full-breathing mode $\mathrm{O}_{B}^{X}$, at the the $X$ point of the $\mathrm{BZ}$ where the $\mathrm{O}_{x}$ and $\mathrm{O}_{y}$ ions vibrate in phase along the bonds against the silent $\mathrm{Cu}$ ion [1] nonlocal EPI generates CF's at the $\mathrm{Cu}$ and we obtain an electronic charge transfer from that $\mathrm{Cu}$ ion where the bonds are compressed to the $\mathrm{Cu}$ where the bonds are stretched [20]. While in case of the HBM the charge stripes point along the $x$ - or $y$-direction, respectively, for $\mathrm{O}_{B}^{X}$ the stripes point along the diagonals in the $\mathrm{CuO}$ plane.

The self-consistent changes of the potential felt by an electron due to nonlocal EPI also have been calculated for the OBSMs and yield a strong coupling of the order $100 \mathrm{meV}$
[19]. It should be remarked that such a strong nonlocal EPI associated with the OBSMs, which can reliably be calculated in the adiabatic approximation, is further enhanced by an order of magnitude because of the poorly screened long range Coulomb interaction and the slow charge dynamics for the polar modes from the nonadiabatic sector of phase space around the $c$-axis where phonon-plasmon mixing is relevant [19].

In Figures 3(a) and 3(b), we present the calculated results for the nonlocally induced charge redistribution of the HBM for the insulating and the pseudogap state, which should be compared to the corresponding results for the optimal doped state in Figure 2(b). First of all we find for all electronic states dynamical stripes along the bond direction excited by the nonlocal EPI being simultaneously responsible for the corresponding phonon softening in Figure 1. This is quite interesting because dynamic charge inhomogeneities of this type may be considered as precursors of static charge stripe order as recently observed in $\mathrm{La}_{2-x} \mathrm{Ba}_{x} \mathrm{CuO}_{4}$, in a broad range of doping around $x=1 / 8$ with $0.095 \leq x \leq 0.155$ [21] or in $\mathrm{YBa}_{2} \mathrm{Cu}_{3} \mathrm{O}_{y}$, respectively, [22].

To the best of our knowledge in overdoped samples static stripe order has not been observed so far. This would mean that the pseudogap in the underdoped state may be required to nucleate dynamic stripes. In our approach this implies that for the emergence of static stripe order the incompressibility of the Cu3d orbitals seems to be essential. The latter brings about a separation of mobile holes populating the compressible $\mathrm{O} 2 p$ states and regions with local Mott- and spin correlations related to the insulator-like $\mathrm{Cu} 3 d$ states. Such stripes producing the onset of a new periodicity that breaks translational and rotational symmetry also may help to 


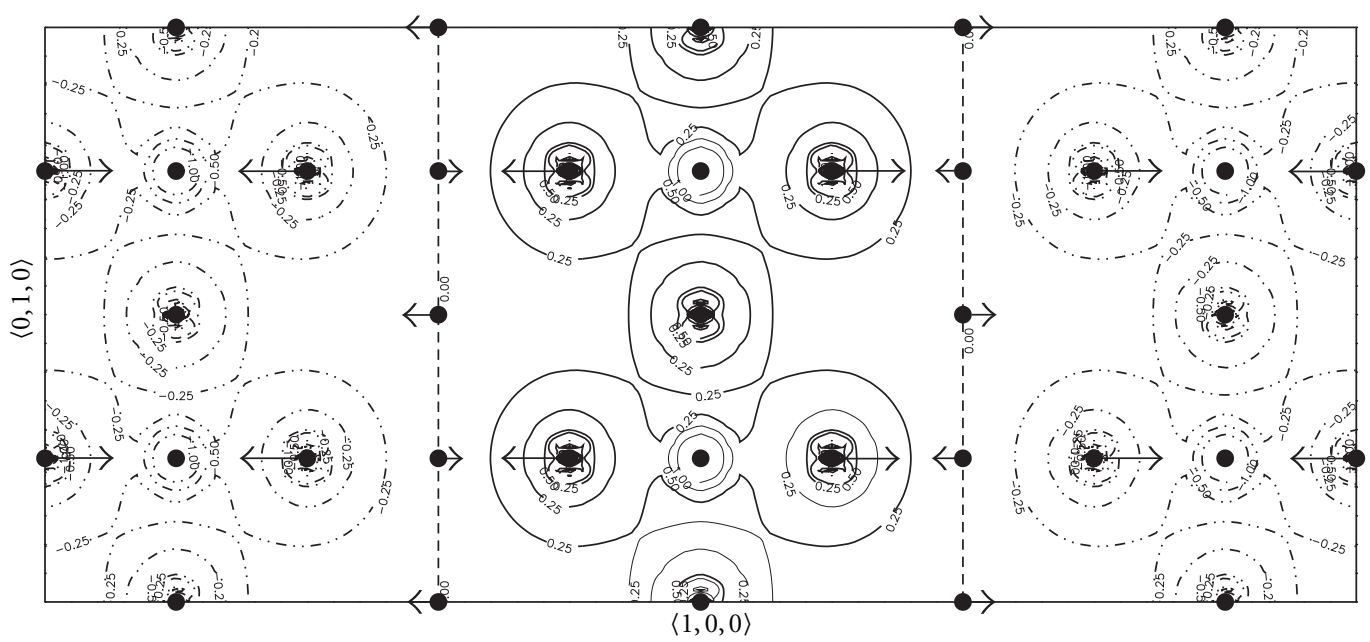

FIGURE 4: Same as Figure 2 but for the nonlocal part of the charge response $\delta \rho$ for $\Delta_{1} / 4$ in the optimally doped state.

trigger a reconstruction of the large hole Fermi surface (FS) into small pockets as detected by quantum oscillation measurements in underdoped $\mathrm{YBa}_{2} \mathrm{Cu}_{3} \mathrm{O}_{6+x}$, see for example, [23] and references therein. Also from an energetic point of view, our modeling of the pseudogap state is susceptible for the tendency to establish broken symmetry states with holerich compressible and hole-poor incompressible regions. In general terms, lowering of the kinetic energy occurs in the compressible metallic regions and lowering of the interaction energy is possible by antiferromagnetic spin correlations and the Coulomb repulsion between the electrons in the region of the incompressible insulator-like Cu3d states. Altogether, in the pseudogap state the doped holes and the superexchangecoupled spins arrange themselves in a cooperative way to promote both charge mobility and locally antiferromagnetic correlations.

Comparing the stripe patterns of the insulating and underdoped state with that of the more conventional metallic optimal and overdoped state (not shown) with a large FS as the locus of the crucial low energy excitations, we find a relatively small contribution of the $\mathrm{O} 2 p$ orbitals degrees of freedom to the density redistribution in the latter case. The weight of the $\mathrm{O} 2 p$ contribution is significantly enhanced in the insulating and pseudogap state, see Figures 3(a) and 3 (b) reflecting in particular the importance of the $\mathrm{O} 2 p$ orbital in the pseudogap state. Holes in the pseudogap state primarily populate the $\mathrm{O} 2 p$ orbitals, while the holes in the more conventional metallic state gain more and more Cu3d character by an increased hybridization as can be seen from a large PDOS for Cu3d leading to a dominating contribution of the charge redistribution at the $\mathrm{Cu}$ ion where the charge response is now metallic, too. See also [24] where it is investigated how the nature of the hole state evolves with doping. Overall, the stripe pattern is more delocalized in the metallic state and localization increases towards the insulating state via the pseudogap state.

In the overdoped state, a growing importance of the $\mathrm{Cu} 4 s$ orbital and accordingly an increase of the Cu $4 s$ element of the polarizability matrix leads to an enhanced softening of the anomalous OBSMs, see Figure 1. The corresponding calculated dynamical stripe pattern is very similar to that of the optimally doped state in Figure 2(b) but somewhat more delocalized because of the increased influence of the more delocalized contribution due to the Cu4s orbital.

From our calculations it becomes obvious that a multiorbital approach is needed that includes besides $\mathrm{Cu} 3 d$ at least $\mathrm{O} 2 p$ orbitals and for the optimally doped and overdoped state in addition $\mathrm{Cu} 4 s$ orbitals in order to give a reliable representation of phonon dynamics and charge response in the cuprates. Single-band models for the $\mathrm{CuO}$ plane believed by many people to describe the cuprates are not sufficient, in particular in context with the discussion of stripes. Altogether, in the optimal and overdoped state increasing of doping enhances the hybridization between $\mathrm{Cu} 3 d, \mathrm{Cu} 4 s$, and $\mathrm{O} 2 p$ orbitals, respectively, with a dominant weight for $\mathrm{Cu} 3 d$. This state becomes compressible metallic with a large PDOS at $\varepsilon_{F}$. So, the particle number fluctuations that characterize the different competing ground states in our model are enhanced as compared to the ground state in the pseudogap regime. Here the hybridization is weaker due to the incompressible insulator-like $\mathrm{Cu} 3 d$ orbital with a vanishing PDOS at $\varepsilon_{F}$.

Translational symmetry-breaking models of the electronic structure of the $\mathrm{CuO}$ plane generated by charge density wave order of a certain wavevector are discussed in the literature and used to characterize Fermi surface reconstructions of a large FS into small pockets, for recent work see $[25,26]$. From scanning tunneling microscopy (STM), neutron diffraction experiments, and nuclear magnetic resonance measurements, the ordering in many cases occurs with a period of about 4 lattice constants [26]. This corresponds to the wavevector $\pi / 2 a(1,0,0)$ of the $\Delta_{1} / 4$ mode. Therefore, it is meaningful to calculate the nonlocally induced charge redistribution also for this mode. The result for the optimal doped state is shown in Figure 4.

We again obtain a stripe-like charge pattern with translational symmetry in the $(0,1,0)$ direction but a four-unit cell repeat distance along the orthogonal direction $(1,0,0)$. 
Contrasting this result with the charge stripe pattern as found for the HBM in Figure 2(b), the stripes for $\Delta_{1} / 4$ are expanded over the whole unit cell, because the CFs do not vanish at the sites of the moving oxygens, unlike $\Delta_{1} / 2$. However, the amplitudes of the CFs are considerably smaller. According to our calculation of the eigenvectors, this is caused by the strongly reduced bond-stretching amplitude of the oxygen ions in the plane for $\Delta_{1} / 4$, while the displacement of the apex oxygen ions is strongly enhanced in the $\Delta_{1} / 4$ mode as compared with the HBM.

In summary, we have applied our modeling of the doping-dependent electronic states of the cuprates to the calculation of the phonon-induced charge inhomogeneities nonlocally induced by the $\Delta_{1} / 2$ and $\Delta_{1} / 4$ mode in $\mathrm{LaCuO}$. The calculations have been performed for the insulating-, pseudogap, and the more conventional metallic state. The compressibility is used as a primary term to characterize the competing ground states controlled by doping. The strong nonlocal EPI of CF type excites in all cases investigated dynamical charge stripes along the $\mathrm{CuO}$ bonds. Differences in orbital character of the charge response in the various states are discussed at hand of the stripe patterns. Experimentally, charge stripe order has been observed in a broad range of doping in $\mathrm{La}_{2-x} \mathrm{Ba}_{x} \mathrm{CuO}_{4}$ and other cuprates, which supports the point of view that dynamical charge stripes generated by certain strongly coupling OBSM may be considered as precursors of static stripe order at least outside the overdoped regime. Such a charge order breaks translational symmetry and may enforce a reconstruction of a larger hole FS into small pockets.

The interplay between the reduced particle density fluctuations related to the insulator-like behaviour of the localized atomic-like $\mathrm{Cu} 3 d$ orbital that remains incompressible for low enough $p$-doping and on the other hand the compressible, metallic $\mathrm{O} 2 p$ orbitals appears to play an important role for the pseudogap state and the tendency to charge ordering in the cuprates.

\section{References}

[1] C. Falter, T. Bauer, and F. Schnetgöke, "Modeling the electronic state of the high- $T_{c}$ superconductor $\mathrm{LaCuO}$ : phonon dynamics and charge response," Physical Review B, vol. 73, no. 22, Article ID 224502, 2006.

[2] T. Bauer and C. Falter, "Microscopic modeling of phonon dynamics and charge response in NdCuO," Physical Review B, vol. 77, no. 14, Article ID 144503, 21 pages, 2008.

[3] A. Svane, "Electronic structure of $\mathrm{La}_{2} \mathrm{CuO}_{4}$ in the self-interaction-corrected density-functional formalism," Physical Review Letters, vol. 68, no. 12, pp. 1900-1903, 1992.

[4] M. Vojta, "Stripes and electronic quasiparticles in the pseudogap state of cuprate superconductors," Physica C, vol. 481, pp. 178-188, 2012.

[5] Y. Kohsaka, C. Taylor, K. Fujita et al., "An intrinsic bond-centered electronic glass with unidirectional domains in underdoped cuprates," Science, vol. 315, no. 5817, pp. 1380-1385, 2007.

[6] L. Pintschovius, "Electron-phonon coupling effects explored by inelastic neutron scattering," Physica Status Solidi B, vol. 242, no. 1, pp. 30-50, 2005.
[7] H. Uchiyama, A. Q. R. Baron, S. Tsutsui et al., "Softening of $\mathrm{Cu}-\mathrm{O}$ bond stretching phonons in tetragonal $\mathrm{HgBa}_{2} \mathrm{CuO}_{4+\delta}$," Physical Review Letters, vol. 92, no. 19, pp. 197005-1, 2004.

[8] J. Graf, M. D’Astuto, C. Jozwiak et al., "Bond stretching phonon softening and kinks in the angle-resolved photoemission spectra of optimally doped $\mathrm{Bi}_{2} \mathrm{Sr}_{1.6} \mathrm{La}_{0.4} \mathrm{Cu}_{2} \mathrm{O}_{6+\delta}$ superconductors," Physical Review Letters, vol. 100, no. 22, Article ID 227002, 2008.

[9] L. Pintschovius and W. Reichhardt, "Neutron scattering in layered copper-oxide superconductors," in Physics and Chemistry of Materials With Low Dimensional Structures, A. Furrer, Ed., vol. 20, Kluwer Academic Publisher, Dordrecht, The Netherlands, 1998.

[10] L. Pintschovius and M. Braden, "Anomalous dispersion of LO phonons in $\mathrm{La}_{1.85} \mathrm{Sr}_{0.15} \mathrm{CuO}_{4}$," Physical Review B, vol. 60, no. 22, pp. R15039-R15042, 1999.

[11] W. Reichardt, "Cu-O bond-stretching vibrations in $\mathrm{YBa}_{2} \mathrm{Cu}_{3} \mathrm{O}_{7}$ studied by inelastic neutron scattering," Journal of Low Temperature Physics, vol. 105, no. 3-4, pp. 807-812, 1996.

[12] T. Fukuda, J. Mizuki, K. Ikeuchi, K. Yamada, A. Q. R. Baron, and S. Tsutsui, "Doping dependence of softening in the bondstretching phonon mode of $\mathrm{La}_{2-x} \mathrm{Sr}_{x} \mathrm{CuO}_{4}(0 \leq x \leq 0.29)$," Physical Review B, vol. 71, no. 6, Article ID 060501, 2005.

[13] L. Pintschovius, D. Reznik, and K. Yamada, "Oxygen phonon branches in overdoped $\mathrm{La}_{1.7} \mathrm{Sr}_{0.3} \mathrm{Cu}_{3} \mathrm{O}_{4}$," Physical Review $B$, vol. 74, no. 17, Article ID 174514, 2006.

[14] R. J. McQueeney, J. L. Sarrao, P. G. Pagliuso, P. W. Stephens, and R. Osborn, "Mixed lattice and electronic states in hightemperature superconductors," Physical Review Letters, vol. 87, no. 7, Article ID 077001, 4 pages, 2001.

[15] M. D’Astuto, P. K. Mang, P. Giura et al., "Anomalous dispersion of longitudinal optical phonons in $\mathrm{Nd}_{1.86} \mathrm{Ce}_{0.14} \mathrm{CuO}_{4+\delta}$ determined by inelastic X-ray scattering," Physical Review Letters, vol. 88, no. 16, Article ID 167002, 4 pages, 2002.

[16] M. D’Astuto, P. K. Mang, P. Giura et al., “Electron-phonon interaction in $\mathrm{N}$-doped cuprates: an inelastic X-ray scattering study," International Journal of Modern Physics B, vol. 17, no. 4-6 I, pp. 484-492, 2003.

[17] M. Braden, L. Pintschovius, T. Uefuji, and K. Yamada, "Dispersion of the high-energy phonon modes in $\mathrm{Nd}_{1.85} \mathrm{Ce}_{0.15} \mathrm{CuO}_{4}, "$ Physical Review B, vol. 72, Article ID 184517, 10 pages, 2005.

[18] T. Bauer and C. Falter, "Dynamic charge inhomogeneity in cuprate superconductors," Journal of Physics Condensed Matter, vol. 22, no. 14, Article ID 142201, 2010.

[19] T. Bauer and C. Falter, "Impact of dynamical screening on the phonon dynamics of metallic $\mathrm{La}_{2} \mathrm{CuO}_{4}$," Physical Review $B$, vol. 80, no. 9, Article ID 094525, 2009.

[20] C. Falter, "Phonons, electronic charge response and electronphonon interaction in the high-temperature superconductors," Physica Status Solidi B, vol. 242, no. 1, pp. 78-117, 2005.

[21] M. Hücker, M. V. Zimmermann, G. D. Gu et al., "Stripe order in superconducting $\mathrm{La}_{2-x} \mathrm{Ba}_{x} \mathrm{CuO}_{4}(0.095 \leq x \leq 0.155)$," Physical Review B, vol. 83, Article ID 104506, 16 pages, 2011.

[22] T. Wu, H. Mayaffre, S. Krämer et al., "Magnetic-field-induced charge-stripe order in the high-temperature superconductor $\mathrm{YBa}_{2} \mathrm{Cu}_{3} \mathrm{O}_{y}$," Nature, vol. 477, no. 7363, pp. 191-194, 2011.

[23] D. LeBoeuf, N. Doiron-Leyraud, B. Vignolle et al., "Lifshitz critical point in the cuprate superconductor $\mathrm{YBa}_{2} \mathrm{Cu}_{3} \mathrm{O}_{y}$ from high-field Hall effect measurements," Physical Review B, vol. 83, Article ID 054506, p. 14, 2011.

[24] Y. Sakurai, M. Itou, B. Barbiellini et al., "Imaging doped holes in a cuprate superconductor with high-resolution compton scattering," Science, vol. 332, no. 6030, pp. 698-702, 2011. 
[25] N. Harrison and S. E. Sebastian, "Protected nodal electron pocket from multiple-Q ordering in underdoped high temperature superconductors," Physical Review Letters, vol. 106, no. 22, Article ID 226402, 2011.

[26] H. Yao, D.-H. Lee, and S. Kivelson, "Fermi-surface reconstruction in a smectic phase of a high-temperature superconductor," Physical Review B, vol. 84, no. 1, Article ID 012507, 2011. 

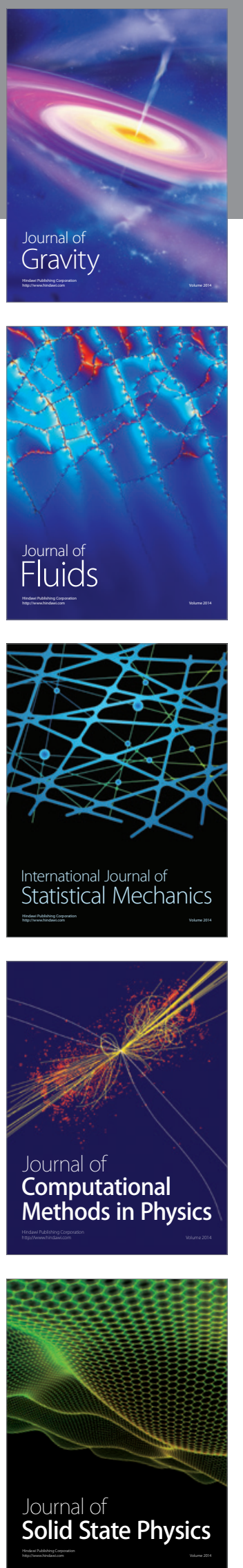

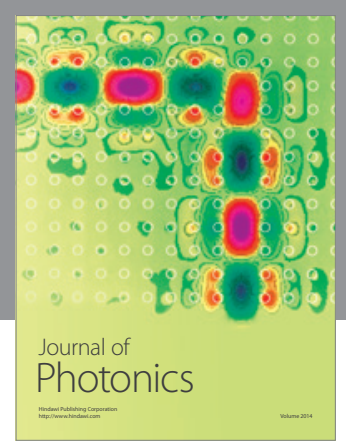

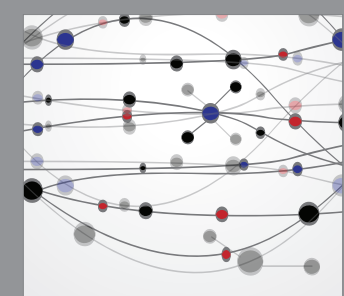

The Scientific World Journal
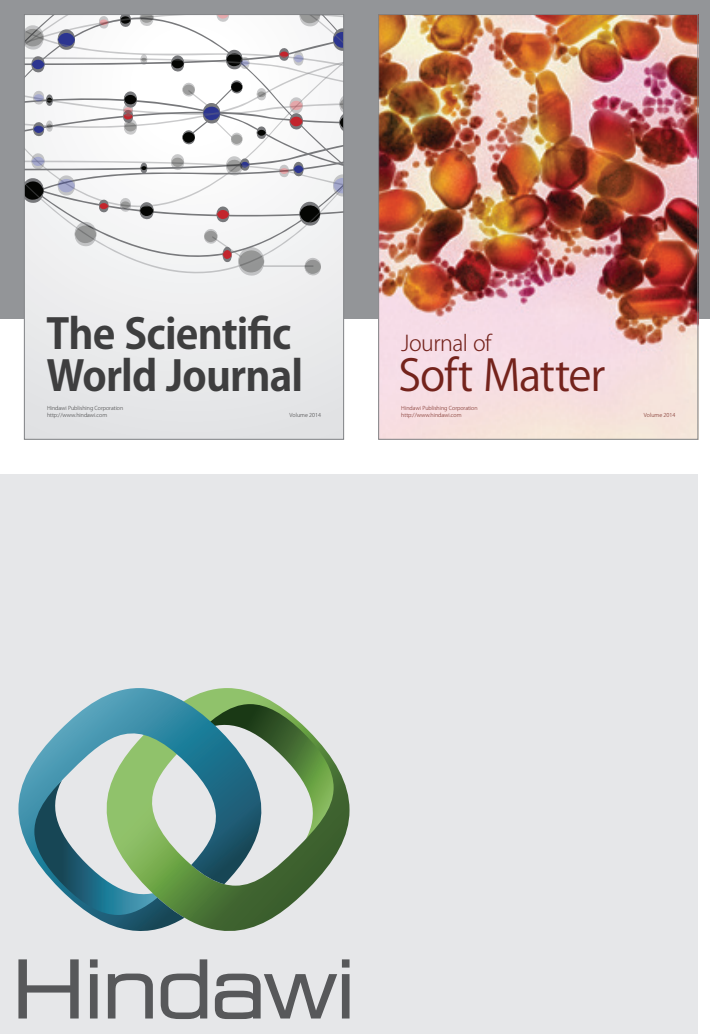

Submit your manuscripts at

http://www.hindawi.com
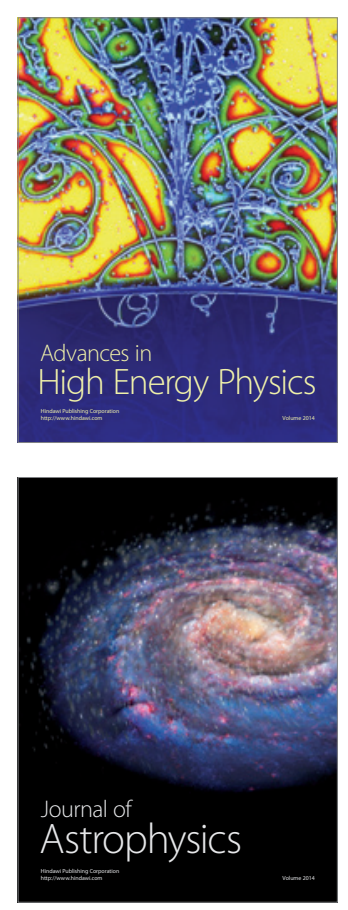
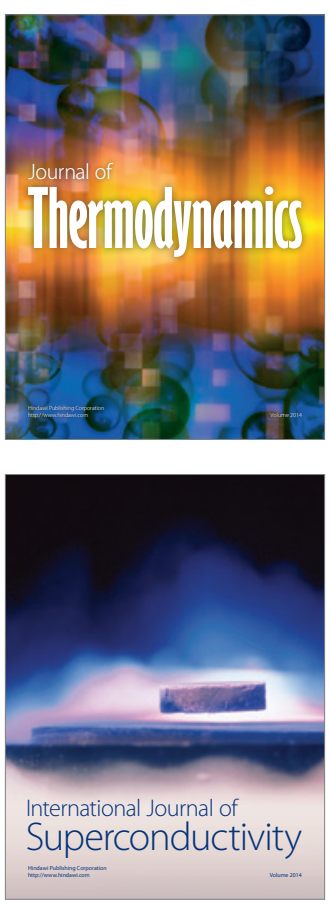
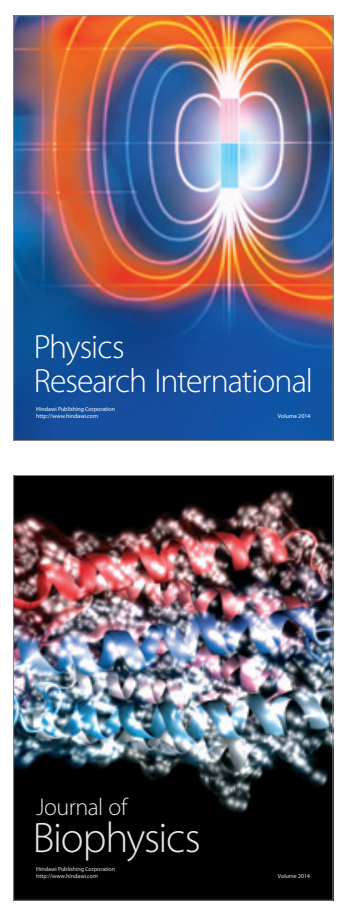
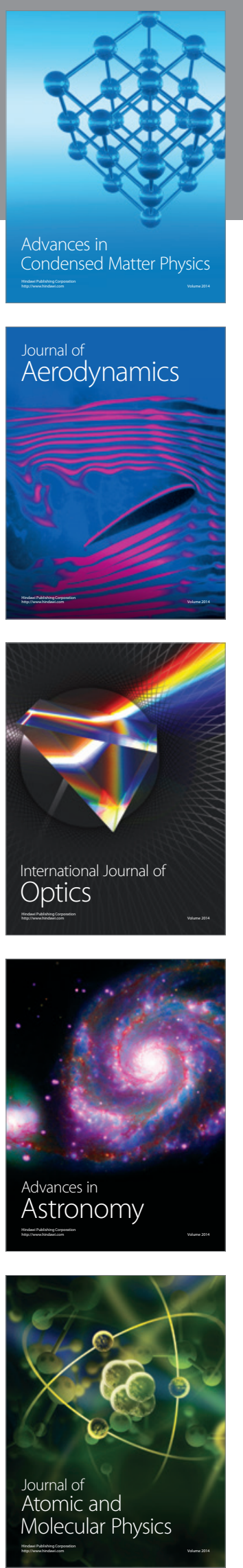